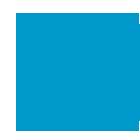

\title{
Der Dialog ist in der Krise eines der stärksten Mittel im Unterricht und in der Führung
}

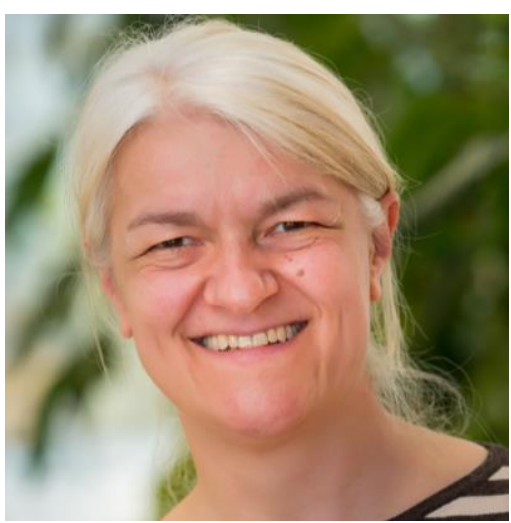

Foto (zvg.): Gabriele Hetzmannseder
Frau Mag. ${ }^{a}$ Gabriele Hetzmannseder war ca. 15 Jahre lang HABITGeschäftsführerin, Mitbegründerin der Basalen Förderklassen in Wien und wirkte in zahlreichen Funktionen im österreichischen Behindertenbereich. Mit Gabriele Hetzmannseder verbinde ich Professionalität, eine motivierende Zielstrebigkeit, Durchsetzungs- fähigkeit - vor allem für jene Menschen, für die sie verantwortlich war - ein großes Fachwissen, ein breites Erfahrungsspektrum im Sozialbereich und der Sonderpädagogik und jene Form von Reformbewusstsein, die es für die (Weiter)Entwicklung der großen Arbeitsfelder der Behindertenpädagogik benötigt. Sie gibt uns einen kurzen Einblick in die Entstehungsgeschichte der Basalen Förderklassen in Wien.

\section{„..weg vom medizinischen Modell (...) hin zu einem sozialen Modell“}

\section{Sie haben einen langjährigen Überblick über die Entwicklungen im Behindertenbereich - wie war Ihr persönlicher Werdegang und welche Meilensteine sehen Sie aus heutiger Perspektive?}

Gabriele Hetzmannseder: Mein persönlicher Einstieg in das Berufsfeld war die Arbeit in einer Wohngemeinschaft für Menschen mit Behinderung die im Zuge der Ausgliederung aus einer psychiatrischen, beziehungsweise pflegerischen Einrichtung aufgebaut wurde. Dies war der Beginn eines Paradigmenwechsels, weg vom medizinischen Modell, bei dem medizinische Sichtweisen und Diagnosen mit defizitorientiertem Denken und damit letztlich auch die „Fragmentierung des Menschen“ im Vordergrund standen, hin zu einem sozialen Modell, das die Behinderung eines Menschen nicht als Krankheit, sondern in einem ganzheitlichen und wertfreien Kontext sieht. In dieser Betrachtungsweise sind die Grenzen zwischen einer Behinderung und Nicht-Behinderung oft fließend, eine Merkmalsnennung oder Zuschreibung muss nicht zwangsläufig erfolgen. Mit dem sozialen Modell kam es auch zu einer verstärkten Hinwendung zur Integration und aktiven Teilhabe in allen gesellschaftlichen Lebensbereichen. Beispielsweise wurde diese „Ausgliederung“ mit dem Bemühen begonnen, Menschen mit einer Behinderung aus den Heimstrukturen 
und den Pflegeeinrichtungsstrukturen in spitalsähnlichen Betreuungsformen „herauszuholen“ und in anfangs noch recht großen - Wohngemeinschaften und später in immer kleiner werdenden Wohneinheiten in Form eines selbstbestimmten Wohnens zu begleiten. Dazu gehören natürlich auch Tagesstrukturen - den jeweiligen, individuellen Bedürfnissen entsprechend. Im Schulbereich hat dies in Folge bedeutet, dass die Forderung bekräftigt wurde, für alle Schüler*innen im schulpflichtigen Alter eine geeignete Form der Beschulung zu schaffen. Neben dem Versuch und dem primären Verständnis einer integrativen Teilhabe am Regelschulsystem wurde zusehend der Ruf nach einer spezialisierten Form der Beschulung für jene Schüler*innen laut, die eine sehr betreuungsintensive Form des Unterrichts benötigten. Hier knüpften 1992 die Basalen Förderklassen ${ }^{1}$ mit ihrem Konzept an.

\section{Wurden Anfang der 90er Jahre - nicht zuletzt aufgrund Ihrer Initiative und Mitarbeit - die Basalen Förderklassen ins Leben gerufen?}

Richtig, damals war das große Ziel des Landesschulinspektors für Sonderpädagogik in Wien die Zahl der von der Schulpflicht befreiten Kinder zu reduzieren. Dank der eingangs beschriebenen Sichtweise, das heißt weg vom Zugang „Behinderung als Krankheit“ und der schrittweisen „Entlassung aus der medizinischen Zuständigkeit“ wurde auch der Schulbesuch neu gedacht. Zum Beispiel, dass ein Schulweg für ein mehrfach schwerstbehindertes und pflegebedürftiges Kind im Sinne der Teilhabe auch zumutbar und damit vertretbar ist - ausgehend von den Heilstättenschulen in den Spitälern. Das hat seitens der Ärzteschaft zu Beginn auch viele Bedenken hervorgerufen. Die ersten Erfahrungen haben aber gezeigt, dass es für die Kinder und Jugendlichen eine absolute Bereicherung ist, eine Schule zu besuchen und auch einen Weg dorthin zurück zu legen.

\section{Die Zahl der schulpflichtbeschränkten Kinder konnte also reduziert weden?}

Ich habe die Basalen Förderklassen die ersten neun Jahre geleitet. Die Zahl der schulpflichtbefreiten Kinder konnte damals auf insgesamt nur noch drei reduziert werden. Das war zunächst natürlich auch ein großes Umdenken für die Angehörigen: von Skepsis (Mein schwerstbehindertes Kind kann bzw. soll den Unterricht besuchen?) bis hin zu Stolz (Mein Kind geht jetzt auch zur Schule!) war zunächst das ganze Spektrum an Emotionen vorhanden.

\section{„Es wurde rasch erkannt, dass es den nötigen Personalschlüssel braucht, um hier professionell arbeiten zu können“}

\section{Mit wie vielen Kindern haben die Basalen Förderklassen gestartet?}

Wir haben mit vier Kindern nach einem Versuch engagierter Pädagog*innen in einer Heilstättenschule gestartet. Sie sind damals nach Deutschland gereist, um sich das Förderkonzept von Dr. Andreas Fröhlich anzusehen und haben dann zurück in Wien mit der Methode der Basalen Stimulation begonnen. Es wurde rasch erkannt, dass es den nötigen Personalschlüssel braucht, um hier professionell arbeiten zu können: d.h. in einer 1:2 Betreuung. Das war mit dem normalen Lehrpersonal nicht längerfristig abzudecken, daher 
wurde über Umwege und zur Aufstockung der Personalressourcen beim Verein der Wiener Sozialdienste eine zweite Person in der Klasse angefragt. Damals wurde ich von den Wiener Sozialdiensten mit der Koordination und dem Aufbau weiterer Basaler Förderklassen einschließlich einer dazugehörigen Nachmittagsbetreuung betraut. Das erste Fachbetreuungspersonal wurde angestellt.

\section{Welche Ausbildung hatte das Lehr- und vor allem das Betreuungspersonal?}

Die Pädagogik stand klar im Fokus aller Bemühungen, durch die Abkehr vom medizinischen Modell, das heißt in weiterer Folge auch von einem primär pflegerischen Aspekt, war eine Ausbildung im Gesundheitswesen kein Muss-Kriterium für das Fachbetreuungspersonal - viel wichtiger waren nun vielfältige, vor allem sonderpädagogische Qualifikationen und Zusatzausbildungen im Bereich der Basalen Stimulation und der Unterstützten Kommunikation sowie generell eine multiprofessionelle Zusammensetzung der Teams in den Basalen Förderklassen. Nach dem ersten Impuls, weg von der „pflegelastigen Gewichtung“ in der Personalauswahl und hin zu einer sonderpädagogischen Akzentuierung, war es in Folge wichtig ein gutes Gleichgewicht im Sinne eines ganzheitlichen pädagogischen Auftrags zu finden. Das musste sich über die Jahre einspielen - am Anfang gab es schon noch die Sichtweise, dass hier schulfremde Personen eine pädagogische Zielausrichtung (mit)bestimmen. Auch die Frage der Separation und Segregation stand zu Beginn im Raum, deshalb versuchte man die Basalen Förderklassen vor Ort in das gesamtpädagogische Konzept der Standorte zu integrieren.

\section{Hatten Sie den Eindruck, dass man in Deutschland in der Schwerstbehindertenpädagogik weiter, bzw. fortschrittlicher war?}

Vielleicht in bestimmten Fachdisziplinen wie der Basalen Stimulation und der Unterstützten Kommunikation (UK). Da war man mit den Konzepten in Deutschland einfach schon viel weiter und einige Jahre voraus. Die importierten Arbeitsweisen wurden jedoch rasch weiterentwickelt und so konnte z.B. das UK-Konzept bei uns auch sehr stark im basalen Bereich angewendet werden und nicht wie in Deutschland, wo es damals primär nach einem Kandidatenmodell ${ }^{2}$ zugänglich gemacht wurde. Basis der Unterstützten Kommunikation und der Interventionsplanung sind der Dialog und die Beachtung körpereigener Kommunikationsformen, im Bereich des somatischen Dialogs konnten wir innovative Konzepte entwickeln. Wir haben bei uns in Österreich auch viel in den Fort- und Weiterbildungsbereich - auch vereins- und schulintern - investiert. Somit konnten allfällige Lücken rasch geschlossen werden.

\section{Wir haben die Kommunikationsförderung im Sozial- und Behindertenbereich „breiter" angelegt?}

Ich habe PPP studiert, daher habe ich mich kontinuierlich mit dem dialogischen Prinzip beschäftigt. Der Dialog, das persönliche Gespräch ist für mich immer die Basis einer Interaktion - erst, wenn ich verstehe, was das Kind - auch nonverbal - sagen möchte, kann das Lernziel gemeinsam gefunden werden, kann ich eine passende Methode anbieten. Aber auch in der Mitarbeiterführung steht für mich der Dialog im Mittelpunkt, denn jede Interaktion sollte auf Augenhöhe erfolgen und Führungskräfte sind auch Vorbilder für diesen Dialog. 


\section{Welche Entwicklungsfelder sehen Sie in der Zukunft der (schulischen) Sonderpädagogik? Gibt es einen Unterschied zwischen den Bundesländern in Österreich der schulischen Konzepte im mehrfachschwerstbehinderten und basalen Bereich?}

Wien und Niederösterreich sind schon aufgrund der geographischen Lage sehr verschieden - das hat natürlich Auswirkungen auf die Anzahl und die Spezialisierung der Einrichtungen. Mir war stets wichtig, dass es hinsichtlich eines funktionierenden Übergangsmanagements auch eine Tagesstruktur gibt, die mit dem methodischen Know-How bei der Förderung im Übergang von „Schule - Beschäftigung und Betreuung" anknüpfen kann. Umgekehrt konnten wir feststellen, dass es große Unterschiede bei der Methodenvielfalt und den Förderkonzepten der Schulstandorte gab - nicht jeder Standort konnte sich spezialisieren, daher gab es natürlich auch Schulabgänger*innen im basalen Bereich die beim Schulaustritt noch wenig Erfahrungen in den Bereichen UK und Basaler Kommunikation gemacht hatten. Ganz wichtig ist im Gesamtkontext immer, so individualisiert wie möglich zu arbeiten. Die Bedürfnisse und Entwicklungspotentiale werden in diesem Bereich sehr klar von den Schüler*innen vorgegeben. Die Basalen Förderklassen waren ein erster Schritt in Richtung Integration in die Gesellschaft, waren zu Beginn aber aufgrund des Konzeptes noch sehr homogen.

Welche schulischen Angebote für Schüler*innen mit Behinderung benötigen wir (z.B. im Bereich der Basalen Förderklassen) parallel zu unserem öffentlichen Regelschulsystem in Hinblick auf Krisenintervention, Standortspezialisierung und/oder die nachschulische Betreuung?

Kleinere Klassen, ein Bekenntnis zur Qualität bei der Ressourcenverteilung, heterogene Klassen, diversitätsbewusste Konzepte, um voneinander lernen zu können und - auf der persönlichen Ebene - kleine, mitunter homogene Lerngruppen, wenn es um eine Spezialisierung in der Förderung geht. Dafür bedarf es einer Profession, der Bereitschaft zum ganzheitlichen Ansatz und der freien Wahl der Methoden. Konkrete Förderpläne und die Relevanz der Lehrpläne sind wichtig, können aber auch viel Druck erzeugen. In der nachschulischen Betreuung gibt es viel weniger Vorgaben von außen, das hilft bei der Realisation von individuellen Konzepten und gemeinsamen Visionen. Grundsätzlich glaube ich für die Zukunft, dass verstärkt auf eine professionelle Ausbildung der Führungskräfte, eine Vermeidung geschlossener Systeme im Schulbereich mithilfe größtmöglicher Transparenz und eine Sensibilisierung des bestehenden Machtgefälles, vor allem in der Interaktion mit schwerstbehinderten Kindern und Jugendlichen, gesetzt werden sollte.

Diese Ausgabe trägt den Titel „Führung in Zeiten der Krise“, welche Kompetenzen können Sie für Führungskräfte und Bildungsverantwortliche nennen, um in Zeiten wie diesen auf die Aufgaben in der Pandemie bestmöglich vorbereitet zu sein?

Ein Teil der Antwort liegt in der zu begrüßenden Schulautonomie - es bedarf allen voran standortspezifischer Lösungen. Für Schulleitungen ist eine gewisse Gestaltungsfreiheit wichtig. Alle Kompetenzen, die mit einer wertschätzenden Dialogführung in Zusammenhang stehen, sind zu begrüßen: ein gemeinsam definierter Handlungsrahmen - klar und breit zugleich -, Feedback geben und einholen, Empathie, Transparenz, am Menschen orientiertes Arbeiten, eine offene Konflikt- und Fehlerkultur etablieren und so 
weiter. Ein Kollegium mit gut gelebter Feedbackkultur ist häufig viel unabhängiger von der unmittelbaren Führungskraft. Das kann in bestimmten Situationen ein Vorteil sein.

Was macht gutes Krisenmanagement aus, wenn man die Verantwortung für eine sensible Klientel (allgemein Schutzbefohlene, Schüler*innen mit Behinderung und/oder einem sonderpädagogischen Förderbedarf (SPF) usw.) trägt?

In der Krise liegt noch mehr Verantwortung bei der Führungskraft. Durch die Krisensituation passiert viel Verunsicherung und es werden Ängste ausgelöst. Das bedarf einer entsprechenden Sensibilität, aber auch klarer und schneller Entscheidungen. Wenn etwas auch anders passieren darf bzw. soll als sonst, braucht es ein gut eingespieltes Team, viel Vertrauen und noch mehr Kommunikation als sonst.

\section{Inklusion und Standortspezialisierung?}

Was wäre aus Ihrer Sicht für die Schulentwicklung wichtig? Wie sehen Sie die aktuelle Debatte zum Thema Inklusion und dem Erhalt der Sonderschulstandorte?

Inklusion und Standortspezialisierung? Die Inklusionsdebatte muss kein Entweder-oder sein, sondern kann auch ein Sowohl-als-auch mit einem mutigen Qualitätsanspruch sein!

Vielen Dank für das Gespräch!

Mit Gabriele Hetzmannseder sprach Matthias Hesse

\section{Anmerkungen}

${ }^{1}$ Die Basalen Förderklassen sind eine für mehrfach behinderte Kinder und Jugendliche angebotene Schulform in Wien, die in dualer Struktur und Kooperation der Bildungsdirektion für Wien und der Wiener Sozialdienste ein Teil des Regelschulsystems wurde.

2 Das Kandidatenmodell beschreibt einen frühen Zugang in der Unterstützten Kommunikation. Personen, die von der Kommunikationsförderung oder einem Kommunikatinshilfsmittel profitieren sollen, müssen über gewisse Grundvorausetzungen, Fähigkeiten und Kompetenzen - vor allem kognitive Voraussetzugen und ein gutes Sprachverständnis - verfügen. Das Kandidatenmodell wurde weitestgehend vom Partizipationsmodell abgelöst. 